\title{
Keyword Index Volume 18 (2014)
}

\begin{tabular}{|c|c|c|c|}
\hline ad creative & 1199 & clustering ensemble & 389 \\
\hline algorithm analysis & 261 & collaborative filtering & 953,973 \\
\hline alternating direction method & 1137 & collaborative tagging & 953 \\
\hline animal feed quality monitoring & 25 & community discovery & 495 \\
\hline APMM stability measure & 389 & component-based algorit & thms \\
\hline apriori algorithm & 637 & concept drift detection & 337 \\
\hline association analysis & 739 & conic quadratic program & ming \\
\hline association mining & 637 & consensus matrix & 531 \\
\hline association rule mining & $217,243,479$ & constrained learning & 927 \\
\hline attribute reduction & 429 & constraint-based mining & 837 \\
\hline attribute selection & 819 & continuous optimization & 79 \\
\hline automated algorithm design & 63 & convex optimization & 1137 \\
\hline automated campaign managem & 1199 & convexity & 79 \\
\hline & & cooperative learning & 511 \\
\hline back-propagation neural netwol & 449 & Cophenetic correlation c & oefficient \\
\hline Bayesian network & 157,281 & credit assignment & 511 \\
\hline Bayesian network classifier & 3 & critic learning & 511 \\
\hline belief clustering & 409 & & \\
\hline biclustering & 837 & data analysis & 1013 \\
\hline big data & 117 & data and knowledge visu & ralization \\
\hline binary decision diagram & 889 & data generation & 761 \\
\hline bitrate estimation & 465 & data mining & $531,583,609,637,697$, \\
\hline byte-level n-gram & 677 & & $793,1013,1109,1177$ \\
\hline & & data privacy & 365 \\
\hline chance threshold & 243 & data set characteristics & 449 \\
\hline change detection & 181 & data stream & 181,337 \\
\hline churn prediction & 3,95 & decision tree & 63,857 \\
\hline class imbalance & 203,1089 & dendrogram & 547 \\
\hline classification $63,95,203,697$ & $7,739,1089,1109$ & density estimation & 157 \\
\hline classifiers & 911 & description matrix & 547 \\
\hline cluster cohesion & 409 & discrete & 583 \\
\hline cluster evaluation & 389 & distributed optimization & 1137 \\
\hline cluster separation & 409 & divergence & 25 \\
\hline clustering & $137,181,479,531$ & diversity & 1067 \\
\hline & $547,793,857,973$ & dynamic clustering & 409 \\
\hline clustering approach & 1109 & & \\
\hline clustering differential privacy & 583 & eigen-space update & 927 \\
\hline
\end{tabular}


encoder rate control

ensemble learning

episode mining

event sequences

evolutionary algorithm

evolutionary search

expertness framework

extended evidence accumulation clustering

95,857,
clustering

feature extraction

feature reweighting

feature selection

feature supervision

fisher exact test

frequent item set mining

frequent itemsets

frequent pattern mining

frequent positive and negative itemset

function approximation

fuzzy clustering

fuzzy K-nearest neighbor

fuzzy rough set

Gaussian process

gene expression data

gene selection

gene-function prediction

genetic algorithms

genetic clustering algorithms

Google AdWords

graph anonymization

Haar wavelet transform

harmfulness

hierarchical clustering

hierarchical clustering combination

hierarchical multi-label classification

hierarchy

high dimension

Hoeffding bound

Hungarian method

hyperspectral band selection

hyperspectral image cubes

imbalanced data
465,927

561

319

561

243

997

1027

637

1049

1153

157,1153

429

429

319

837

739

717

1199

819

1199

365

583

203

547

547

717

531

157

337

531

25

25

95 imbalanced ratio

203

1013

instance selection $\quad 429$

integer programming 43

integrative 739

interactive clustering 561

interactive feature selection $\quad 561$

interactive multidimensional data visualization

$k$ value prediction model

449

keyword selection

1199

K-nearest neighbor

429

$\mathrm{kNN}$ classifier

677

$k$-NN classification algorithm

449

label dependency $\quad 911$

land covers $\quad 305$

large datasets $\quad 793$

LDA 1067

length principle $\quad 889$

linear discriminant analysis $\quad 927$

linear models $\quad 79$

local discriminative subspaces $\quad 319$

local ICA 157

lognormal distribution $\quad 1067$

log-normality 1067

loss function 697

machine learning 697

matrix completion 1137

maximal frequent $\mathrm{U} 2$ patterns $\quad 653$

maximum profit criterion 3

mean shift outliers 79

meta learning 95, 261

methodologies and tools $\quad 873$

metric embedding $\quad 365$

microarray data 739

minimum description 889

mining methods and algorithms $\quad 609$

missing data 1177

missing-data mechanism $\quad 1177$

model validation $\quad 873$

model-based techniques $\quad 973$

models $\quad 79$ 
MU2P-Miner

multi-agent systems

multiclass imbalanced datasets

multilabel classification

multiple minimum supports

multivariate microaggregation

nave Bayes

nearest neighbor classifier

negative association rule

negative itemset

network flow

news relation discovery

$\mathrm{NMF}$

nodes importance evaluation

noise

novelty detection

one-class classification

online advertising

online learning

optimization

outlier detection

outlier observation

parallel episodes

parallel spike trains

pattern mining

pay-per-click advertising

prediction intervals

privacy preservation

probabilistic

quality evaluation

questionnaire data

random subspace method

rank-order mismatch

rare frequent itemsets

RBF networks

recommender systems

recursive feature elimination

reflectance analysis

region-based ranking

regression
653 reinforcement learning

1153

511 requent trajectory pattern 637

1109 reusable components 63

911,1137 robotic soccer simulation 973

1027 rough set 429

819 rule ensembles 857

261 sampling approach 1109

319 sampling method 1089

243, 1049 satellite image classification 305

1049 scatter matrix based class separability

43 measure 203

217 scatter matrix update $\quad 927$

495 segmentation 305

495 semi-supervised learning 857

511 sensitive information 281

181 sensitive XML association rules 281

sensitivity 1067

889 sequential data $\quad 137$

1199 sequential pattern 1013

927 sessionization 43

697 similarity upper approximation $\quad 137$

1049 sliding window 337

79 social tagging 953

spatial data analysis $\quad 305$

997 spatial/temporal databases $\quad 609$

997 spike synchrony $\quad 997$

761 sporadic rules 243

1199 stability 1067

873 stability indices 531

281 stability-based algorithm 531

911 star-coordinate models 117

state abstraction $\quad 1153$

761 statistics $\quad 79$

479 support vector data description 95

support vector machines 95, 717, 973

1089 support vector regression 465

217 tabular data anonymization $\quad 365$

261 temporal neighborhoods 609

953 text categorization 677

25 text classification 911

25 text mining 217

217 textual advertising 1199

873 time series $\quad 793$ 
1238

topic models

transfer learning

Transferable Belief Model (TBM)

transition regions identification

U2GenMax

U2MAFIA

uncertainty

univariate uncertain data

unsupervised learning

user supervision

utility mining
Keyword Index Volume 18 (2014)

1067 validity measure 531

1153 visual cluster analysis 117

409

305 wavelet transforms 583

web server logs 43

653 web usage data 137

653 web usage mining 43

305, 409 web user 43

653 weighted directed network 495

181 Wyner-Ziv (WZ) video coding 465

561

1027 XML

281 